Pramāna, Vol. 23, No. 6, December 1984, pp. 745-749. (C) Printed in India.

\title{
An explanation of the phenomenon of polytypism
}

\author{
S RAMASESHA \\ Solid State and Structural Chemistry Unit, Indian Institute of Science, \\ Bangalore 560012, India

\begin{abstract}
Based on the analogy between polytypes and spin-half Ising chains, polytypes can be considered as different phases of a spin-half Ising system with competing nearest neighbour and next nearest neighbour interactions operating in a single direction. It is known that such an Ising system exhibits extremely rich and complicated phase behaviour. This behaviour is shown to be very similar to the phase behaviour exhibited by polytypes.
\end{abstract}

Keywords. Polytypism; Ising chain; anisotropic next nearest neighbour Ising model.

PACS No. $81.30 \mathrm{Hd} ; 64.70 \mathrm{~Kb}$

\section{Introduction}

Polytypism is a phenomenon exhibited by crystals such as $\mathrm{SiC}, \mathrm{CdI}_{2}$ and $\mathrm{TaS}_{2}$. Different polytypic forms of a substance exhibiting polytypism have the same $a$ and $b$ dimensions of the unit cell but have widely different $c$-dimensions. This phenomenon arises because of the differences in the sequence in which the atomic layers are stacked along the $c$-axis. Some of the striking features of polytypism are (i) the existence of many different types of unidimensional order, (ii) the apparent violation of Gibbs' phase rule owing to the coexistence of a large number of polytypic phases with no discernible differences in stability under identical conditions, (iii) the growth of different polytypic forms in different parts of the same specimen, a feature known as syntactic coalescence and (iv) the existence of varying amounts of disorder in some polytypes. Several theories have been put forth to explain this phenomenon (Verma and Trigunayat 1974), but none explains satisfactorily the above features of polytypism. Recently (Ramasesha and Rao 1977; Uppal et al 1980) attempts to simulate growth of polytypes using the analogy between one-dimensional spin-half Ising chains and polytypes have succeeded in explaining the formation of different polytypes to a certain extent. However, the models used by them have not been able to explain either the existence of a large number of polytypes (e.g. more than a hundred SiC polytypes are known to exist) or the extremely large $c$-dimension of the unit cell (of the order of hundreds of Angstroms) encountered in some polytypes. The purpose of the present note is to discuss the competing interaction model invented by Elliott (1961) and recently studied by Bak (1981), Bak and von Boehm (1980), von Boehm and Bak (1979), Fisher and Selke (1980), Selke and Fisher (1979), and Villain and Gordon (1980), in the context of polytypism. 


\section{The competing interaction model}

Elliott (1961) put forth the competing interaction model to explain the sinusoidal spin ordering observed in some of the rare earth metals, namely, erbium and thulium. This model consists of Ising spins on a simple cubic lattice whose nearest neighbour interaction is ferromagnetic, while next nearest neighbour interaction is antiferromagnetic and the latter exists only along the $z$-direction. The Hamiltonian of this model, also called the anisotropic next nearest neighbour Ising (ANNNI) model can be written as,

$$
\begin{aligned}
H=\sum_{x, y, z} & {\left[J _ { 1 } \left(S_{x, y, z}^{z} S_{x \pm 1, y, z}^{z}+S_{x, y, z}^{z} S_{x, y \pm 1, z}^{z}\right.\right.} \\
& \left.\left.+S_{x, y, z}^{z} S_{x, y, z \pm 1}^{z}\right)+J_{2} S_{x, y, z}^{z} S_{x, y, z \pm 2}^{z}\right]
\end{aligned}
$$

Original theoretical analysis showed that this model displayed a low temperature ordered phase with a sinusoidally varying magnetization along the $z$-direction. The wavevector corresponding to the sinusoidal variation was essentially independent of temperature and determined solely by the ratio $-J_{2} / J_{1}$.

The ANNNI model has recently attracted much attention, theoretically, as it is known to display a Lifshitz point* (Redner and Stanley 1977). Furthermore, it has also been successfully employed in explaining the complicated phase diagram of CeSb which exhibits several different types of stacking of ordered magnetic layers (Fisher et al 1978). The mean field equations of the ANNNI model were numerically solved by Bak and von Boehm $(1979,1980)$. Selke and Fisher (1979) used Monte Carlo technique as well as low temperature expansion technique (Fisher and Selke 1980) to study this model. The salient features of the ANNNI model are that for a given ratio of $-J_{2} / J_{1}$, the equilibrium wave vector, $q$, of the sinusoidally modulated phase varies with temperature as shown in figure 1 . We notice that the temperature range over which a given wavevector of the sinusoidally modulated phase is stable becomes narrow as the temperature increases. The $q-T$ curve is nonanalytic in nature and is known as the 'devil's stair case'. The free energy differences between the different phases at any temperature also are rather small. Figure 2 gives the phase diagram of the ANNNI model. We see that at a given temperature, depending upon the ratio $-J_{2} / J_{1}$, one can have a different sinusoidally modulated phase as the equilibrium phase. The phase diagram becomes extremely complicated near $T_{c}$, the temperature above which the system is paramagnetic. In the grey region of the phase diagram, the stable phases are either the higher order commensurate phases or the incommensurate phases. The incommensurate phases can be described as consisting of commensurate regions separated by domain walls or solitons. The commensurate and incommensurate phases in this region of the phase diagram are separated by chaotic phases (Bak 1981). In $\$ 3$ we discuss the relevance of this phase behaviour in the context of polytypism.

\footnotetext{
* Lifshitz point in the magnetic context is the point in the magnetic phase diagram which separates a uniformly ordered ferromagnetic phase $(q=0)$, a sinusoidally modulated ordered phase $(q=0)$ and a paramagnetically disordered phase.
} 


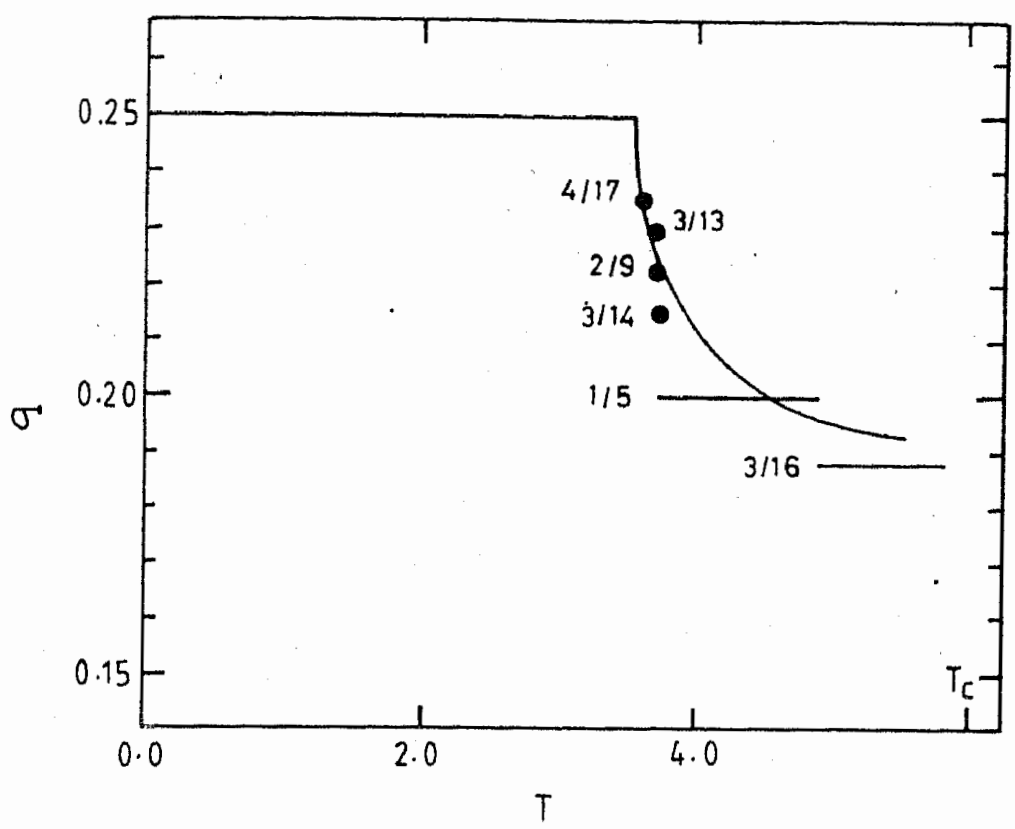

Figure 1. Equilibrium wavevector vs temperature for $J_{2} / J_{1}=-0.7$. The dots and the horizontal lines are results of numerical mean field calculations. Full curve is the result of soliton theory (Bak and von Boehm 1980).

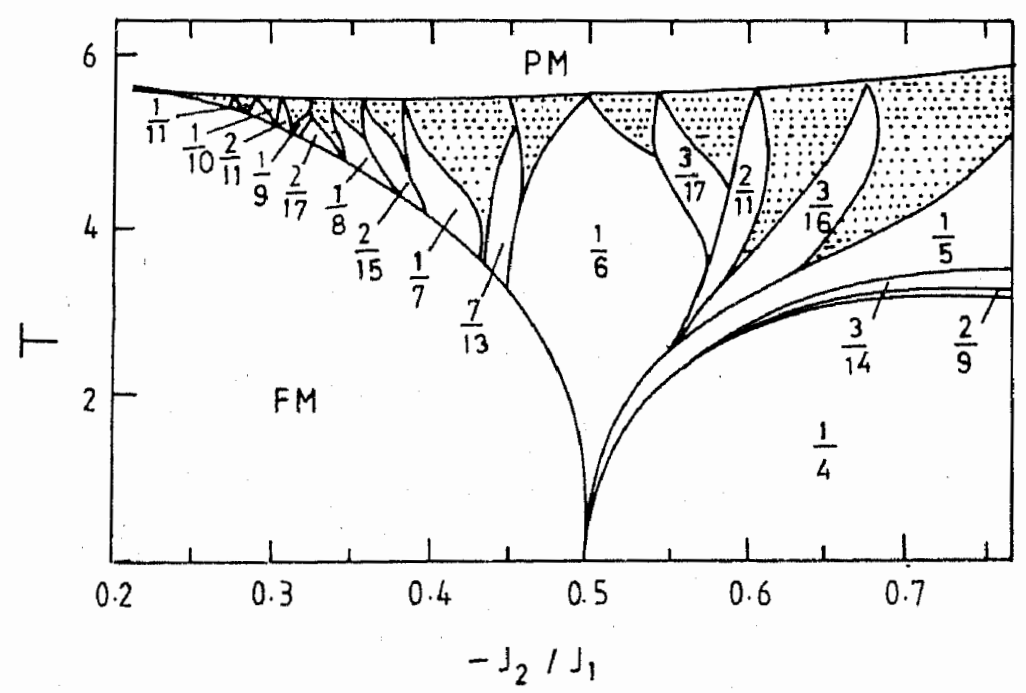

Figure 2. Full phase diagram of the ANNNI model. The dotted region of the diagram consists of commensurate and incommensurate phases separated by chaotic phases (Bak and von Boehm 1980).

\section{Relevance of ANNNI model to polytypism}

In polytypes, a given layer can exist in any one of the two configurations, namely, cubic $(k)$ or hexagonal $(h)$ and a polytype can be uniquely described by specifying the $h k$ sequence of the layers in the unit cell. Similarly, any of the phases of the ANNNI model 
can be uniquely described by giving the spin orientations (either up or down) of all the layers in the unit cell. In the earlier work on polytypes (Ramasesha and Rao 1977; Uppal et al 1980), this analogy between the spin-half Ising systems and polytypes was used to simulate polytypes. The Hamiltonian used for the simulation contained an infinite-range 'ferromagnetic' interaction term competing against short-range 'antiferromagnetic' interaction terms extending up to second nearest neighbours. However, as has been shown by Villain and Gordon (1980), elastic strains and harmonic fields can mediate oscillating indirect interactions between particles (layers) if the direct interactions are not limited to nearest neighbours. Simple potential energy calculations on aggregates of layers (Uppal et al 1980) indeed show that direct inter-layer interactions are not limited to nearest neighbours. Thus, it appears that polytypes are well represented by the ANNNI model. The interactions in the $x-y$ plane in the ANNNI model are necessary only to provide long-range order at non-zero temperature and do not play any significant part in determining the structure of the phases. This role in polytypes could be played by the infinite range "ferromagnetic" interaction between the layers as proposed by Ramasesha and Rao (1977). Even otherwise, polytypes are truly three-dimensional systems and hence the phase diagram predicted by the mean field theory should adequately describe the real situation.

Having qualitatively justified the appropriateness of the ANNNI model to describe polytypism, it is easy to see how all the striking features of polytypism can be fully explained on the basis of the phase diagram of the ANNNI model. In the polytype language, the phase diagram predicts that as the temperature is varied we would scan through an infinity of polytypic phases. Each polytypic phase is stable over a small range of temperature and the wavevector of each stable phase at a given temperature is given by the 'devil's stair case' (figure 1). The many different polytypic forms observed at ordinary conditions of temperature and pressure would then correspond to the metastable states. Since at the time of growth the short-range interactions may fluctuate presumably due to the random motion of the atoms in the layers (Uppal et al 1980) it is possible that at a given temperature many polytypic forms are stable. However, the number of such stable phases reduce to two as the temperature is lowered (figure 2). In any case it is rather difficult to establish experimentally any single polytypic phase as the stable phase for the free energy differences between the phases at any given temperature and pressure are very small-a criterion essential for the existence of a 'devil's stair case'. Thus, ANNNI model explains two of the fundamental properties of polytypism, namely, existence of different types of unidimensional order as well as existence of many polytypic phases with no discernible differences in their stability under identical conditions. Syntactic coalescence can also be explained based on the fact that the temperature range over which a given wave vector is stable becomes extremely narrow as $T_{c}$ is approached. Hence, it is possible that the slight temperature gradient that might exist across the specimen during crystallization could lead to different polytypic orderings in different regions of the specimen. Finally, polytypes with large number of stacking faults could correspond to incommensurate phases (the wave vector $q$ not equal to a rational fraction of the inverse interlayer spacing). It is known that incommensurate phases consist of large regions of the nearest commensurate phase separated by domain walls or solitons. In the polytype context the domain walls or solitons would be the familiar stacking faults. Thus, we see that ANNNI model describes all the important features of polytypism. 


\section{Acknowledgement}

The author is grateful to Dr I Peschel for a useful discussion and U.K. Science Research Council for financial support.

\section{References}

Bak P 1981 Phys. Rev. Lett. 46791.

Bak P and von Boehm J 1980 Phys. Rev. B21 5297

Elliott R J 1961 Phys. Rev. 124346

Fisher M E and Selke W 1980 Phys. Rev. Lett. 441502

Fisher P, Lebech B, Meier G, Rainford B D and Vogt J 1978 J. Phys. C11 345

Ramasesha S and Rao C N R 1977 Philos. Mag. 34827

Redner S and Stanley H E 1977 Phys. Rev. B16 4901

Selke W and Fisher M E 1979 Phys. Rev. B20 257

Uppal M K, Ramasesha S and Rao C N R 1980 Acta Crystallogr. A36 356

Verma A R and Trigunayat G C 1984 Solid state chemistry (ed.) C N R Rao (New York: Marcell Dekker)

Villain J and Gordon M B 1980 J. Phys. C13 3117

von Boehm J and Bak P 1979 Phys. Rev. Lett. 42122 